\title{
Reeh-Schlieder Defeats Newton-Wigner: On alternative localization schemes in relativistic quantum field theory
}

\author{
Hans Halvorson \\ Department of Philosophy, University of Pittsburgh \\ E-mail: hphst1+@pitt.edu
}

November 13, 2018

\begin{abstract}
Many of the "counterintuitive" features of relativistic quantum field theory have their formal root in the Reeh-Schlieder theorem, which in particular entails that local operations applied to the vacuum state can produce any state of the entire field. It is of great interest then that I.E. Segal and, more recently, G. Fleming (in a paper entitled "Reeh-Schlieder meets Newton-Wigner") have proposed an alternative "Newton-Wigner" localization scheme that avoids the Reeh-Schlieder theorem. In this paper, I reconstruct the Newton-Wigner localization scheme and clarify the limited extent to which it avoids the counterintuitive consequences of the Reeh-Schlieder theorem. I also argue that neither Segal nor Fleming have provided a coherent account of the physical meaning of Newton-Wigner localization.
\end{abstract}

\section{Introduction}

Relativistic quantum theory presents us with us a set of peculiar interpretive difficulties over and above the traditional ones of elementary quantum mechanics. For example, while the notion of a "localized object" has a transparent mathematical counterpart in elementary quantum mechanics, 
it appears that not every aspect of our common-sense notion of localization can be maintained in the context of relativistic quantum theory (cf. Malament 1996). Many of the thorny issues involving localization in relativistic quantum field theory have a common formal root in the so-called "Reeh-Schlieder theorem." Thus, it is of particular philosophical interest that I.E. Segal (1964) and, more recently, G. Fleming (2000) have claimed to be able to avoid the Reeh-Schlieder theorem - and thereby its "counterintuitive" consequences - by means of a judicious reworking of the standard association between observables and regions of space.]

I am not convinced, however, that Segal and Fleming's "Newton-Wigner" localization scheme offers any satisfying resolution for the "problem" of localization in relativistic quantum field theory. In particular, the Newton-Wigner localization scheme is not completely immune from the consequences of the Reeh-Schlieder theorem; and neither Segal nor Fleming has offered a conceptually coherent description of the physical meaning of Newton-Wigner localization.

The context of the Reeh-Schlieder theorem is the axiomatic (or algebraic) approach to quantum field theory. This approach singles out a family of postulates that apply quite generally to "physically reasonable" quantum field models, and these postulates are used as a starting point for further structural investigations. One might expect, then, that Segal and Fleming would attempt to undercut the Reeh-Schlieder theorem by questioning one of the assumptions it makes concerning which models are "physically reasonable." However, Segal and Fleming do not discuss the Reeh-Schlieder theorem at this level of generality; rather, their discussion of the Reeh-Schlieder theorem is restricted to a concrete field model, viz., the free Bose field.

I begin then in Section 2 with a brief review of the global structure of the free Bose field model. In Section 3, I present the standard recipe for assigning observables to regions in space, and I explicate the counterintuitive consequences - stemming from the Reeh-Schlieder theorem - of this standard localization scheme. In Section 4, I present the Newton-Wigner localization scheme and show how it "avoids" the counterintuitive consequences of the Reeh-Schlieder theorem. Finally, in Sections 5 and 6, I argue that Reeh-

\footnotetext{
${ }^{1}$ Saunders 1992 provides an extensive discussion of Segal's approach, although with different points of emphasis than the current presentation. Although Fleming $(1996,12)$ appears to dismiss Saunders' comparison of his and Segal's approaches, Fleming's proposal for NW-local algebras (Fleming 2000) (prior to "covariant generalization") is mathematically identical to Segal's (1964) proposal.
} 
Schlieder has the final word against the Newton-Wigner localization scheme.

\section{The free Bose field}

In this section, I briefly review the mathematical formalism for the quantum theory of the free Bose field. Although my presentation differs from Fleming's (2000) in being more abstract and in its emphasis on mathematical rigor, I take it that all parties agree concerning the global structures of the free field model (at least in the absence of measurement interactions). That is, we agree on our answers to the following four questions:

1. What is the state space?

2. What are the observables (i.e., physical quantities)?

3. When no measurements are being made, how does the system evolve in time? In other words, what is the (free) Hamiltonian?

4. What is the ground (i.e., vacuum) state?

Disputes arise only at the level of the local structure of the free field model; e.g., which states are "localized" in this region of space? In this section, I spell out the answers to questions 1-4. In Section 3, I take up questions concerning localization.

Recall that in its heuristic formulation, the free scalar quantum field is described by an "operator-valued field" $\Phi$ on Minkowski spacetime that solves the Klein-Gordon equation

$$
\frac{\partial^{2} \Phi}{\partial t^{2}}+m^{2} \Phi=\nabla^{2} \Phi,
$$

and that satisfies the appropriate (equal-time) canonical commutation relations. As is well-known, however, there are mathematical difficulties with understanding $\Phi$ as an operator-valued function. A more rigorous approachtakes $\Phi$ as an "operator-valued distribution." That is, for each smooth, real-valued test-function $f$ on Minkowski spacetime, $\Phi(f)$ can be defined as an operator on some Hilbert space.

For my purposes here, it will be more convenient to turn to another (mathematically equivalent) representation of the field $\Phi$. Let $C_{0}^{\infty}\left(\mathbb{R}^{3}\right)$ denote 
the vector space of smooth, compactly supported functions from $\mathbb{R}^{3}$ into $\mathbb{R}$, and let

$$
S=C_{0}^{\infty}\left(\mathbb{R}^{3}\right) \oplus C_{0}^{\infty}\left(\mathbb{R}^{3}\right) .
$$

Recall now that a scalar-valued solution $\phi$ of the Klein-Gordon equation is uniquely determined by its Cauchy data (i.e., its values, and the values of its first derivative) at any fixed time. Thus, there is a one-to-one correspondence between elements of $S$ and (a certain subset of) the space of solutions of the Klein-Gordon equation. Moreover, the conserved four-vector current $\phi \overleftrightarrow{\partial}_{\mu} \psi$ gives rise to a symplectic form $\sigma$ on $S$ :

$$
\sigma\left(u_{0} \oplus u_{1}, v_{0} \oplus v_{1}\right)=\int_{\mathbb{R}^{3}}\left(u_{0} v_{1}-u_{1} v_{0}\right) d^{3} \mathbf{x} .
$$

We let $D_{t}$ denote the natural (inertial) symplectic flow on $S$; i.e., $D_{t}$ maps the time-zero Cauchy data of $\phi$ to the time- $t$ Cauchy data of $\phi$. The triple $\left(S, \sigma, D_{t}\right)$ contains the essential information specifying the classical theory of the scalar field of mass $m$.

A representation of the Weyl form of the canonical commutation relations (CCRs) is a mapping $f \mapsto W(f)$ of $S$ into unitary operators acting on some Hilbert space $\mathcal{K}$ such that $W(0)=I$ and

$$
W(f) W(g)=e^{-i \sigma(f, g)} W(f+g) .
$$

I will now sketch the construction of the unique (up to unitary equivalence) "Minkowski vacuum representation" of the CCRs. This construction proceeds in two steps. In first quantization, we "Hilbertize" the classical phase space $S$, and we "unitarize" the classical dynamical group $D_{t}$. More precisely, suppose that $\mathcal{H}$ is a Hilbert space, and that $U_{t}$ is a weakly continuous one-parameter group of unitary operators acting on $\mathcal{H}$. Suppose also that the infinitesimal generator $A$ of $U_{t}$ is a positive operator; i.e., $(f, A f) \geq 0$ for all $f$ in the domain of $A$. If there is a one-to-one real-linear mapping $K$ of $S$ into $\mathcal{H}$ such that

1. $K(S)+i K(S)$ is dense in $\mathcal{H}$,

2. $2 \operatorname{Im}(K f, K g)=\sigma(f, g)$,

3. $U_{t} K=K D_{t}$, 
then we say that the triple $\left(K, \mathcal{H}, U_{t}\right)$ is a one-particle structure over $\left(S, \sigma, D_{t}\right)$. Constructing a one-particle structure over $\left(S, \sigma, D_{t}\right)$ is a mathematically rigorous version of "choosing the subspace of positive frequency solutions" of the space of complex solutions to the Klein-Gordon equation.

If there is a one-particle structure over $\left(S, \sigma, D_{t}\right)$, then it is unique up to unitary equivalence (Kay 1979). That is, suppose that $\left(K, \mathcal{H}, U_{t}\right)$ and $\left(L, \widetilde{\mathcal{H}}, \widetilde{U}_{t}\right)$ are one-particle structures over $\left(S, \sigma, D_{t}\right)$. Then, $L \circ K^{-1}$ extends uniquely to a unitary mapping $V$ from $\mathcal{H}$ onto $\widetilde{\mathcal{H}}$.

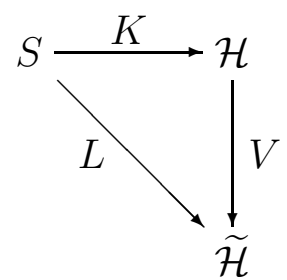

It is also not difficult to see that $V$ intertwines the unitary groups on the respective Hilbert spaces, i.e., $V U_{t}=\widetilde{U}_{t} V$. This uniqueness result can be interpreted as showing that the choice of time evolution in the classical phase space suffices to determine uniquely the (first) quantization of the classical system.

I will construct two (unitarily equivalent) versions of the one-particle structure over $\left(S, \sigma, D_{t}\right)$. First, we may complete $S$ relative to the unique Hilbert space norm in which time-evolution (given by $D_{t}$ ) is an isometry. Specifically, let $H$ denote the linear operator $\left(-\nabla^{2}+m^{2}\right)^{1 / 2}$ on $C_{0}^{\infty}\left(\mathbb{R}^{3}\right)$, and define a real inner-product $\mu$ on $S$ by

$$
\begin{aligned}
\mu\left(u_{0} \oplus u_{1}, v_{0} \oplus v_{1}\right) & =(1 / 2)\left(\left(u_{0}, H v_{0}\right)+\left(u_{1}, H^{-1} v_{1}\right)\right) \\
& =(1 / 2)\left(\int_{\mathbb{R}^{3}} u_{0}\left(H v_{0}\right) d^{3} \mathbf{x}+\int_{\mathbb{R}^{3}} u_{1}\left(H^{-1} v_{1}\right) d^{3} \mathbf{x}\right)
\end{aligned}
$$

Now let $\mathcal{H}_{\mu}$ denote the completion of $S$ relative to the inner-product $\mu .5$ Define an operator $J$ on $\mathcal{H}_{\mu}$ by setting

$$
J\left(u_{0} \oplus u_{1}\right)=-H^{-1} u_{1} \oplus H u_{0},
$$

\footnotetext{
${ }^{2}$ The mathematically rigorous definition of $H$ is as follows: Define the operator $A=$ $-\nabla^{2}+m^{2}$ on $C_{0}^{\infty}\left(\mathbb{R}^{3}\right)$. Then, $A$ is essentially self-adjoint, and the self-adjoint closure $\bar{A}$ of $A$ is a positive operator with spectrum in $\left[\mathrm{m}^{2}, \infty\right)$. Using the functional calculus for unbounded operators, we may define $H=\bar{A}^{1 / 2}$, and it follows that the spectrum of $H$ is contained in $[m, \infty)$.

${ }^{3}$ If $\mathcal{L}^{ \pm}\left(\mathbb{R}^{3}\right)$ denotes the completion of $C_{0}^{\infty}\left(\mathbb{R}^{3}\right)$ relative to the inner product $\left(\cdot, H^{ \pm 1} \cdot\right)$, then $\mathcal{H}_{\mu}=\mathcal{L}^{+}\left(\mathbb{R}^{3}\right) \oplus \mathcal{L}^{-}\left(\mathbb{R}^{3}\right)$.
} 
on the dense subset $S$ of $\mathcal{H}_{\mu}$. Clearly $J^{2}=-I$, i.e., $J$ is a "complex structure" on $\mathcal{H}_{\mu}$. Thus, $\mathcal{H}_{\mu}$ becomes a complex vector space when we define scalar multiplication by $(a+i b) f=a f+J(b f)$, and is a complex Hilbert space relative to the inner-product

$$
\begin{aligned}
(f, g)_{\mu} & =\mu(f, g)+i \mu(J f, g) \\
& =\mu(f, g)+(i / 2) \sigma(f, g) .
\end{aligned}
$$

Finally, it can be shown that $\left[J, D_{t}\right]=0$, so that $D_{t}$ extends uniquely to a weakly continuous one-parameter group of unitary operators (denoted again by $\left.D_{t}\right)$ on the complex Hilbert space $\mathcal{H}_{\mu}$. Therefore, $\left(\iota, \mathcal{H}_{\mu}, D_{t}\right)$, with $\iota$ the identity mapping, is a one-particle structure over $\left(S, \sigma, D_{t}\right)$.

It may not be immediately obvious - especially to those accustomed to non-relativistic quantum mechanics - how to tie the physics of localization to the mathematical structure of the Hilbert space $\mathcal{H}_{\mu}$. (For example, which vectors in $\mathcal{H}_{\mu}$ are localized in a given spatial region?) The Newton-Wigner one-particle structure brings us back to familiar territory by using the space $L^{2}\left(\mathbb{R}^{3}\right)$ as the concrete representation of the one-particle space. In particular, define the mapping $K: S \mapsto L^{2}\left(\mathbb{R}^{3}\right)$ by

$$
K\left(u_{0} \oplus u_{1}\right)=2^{-1 / 2}\left(H^{1 / 2} u_{0}+i H^{-1 / 2} u_{1}\right) .
$$

It is then straightforward to check that the complex-linear span of $K(S)$ is dense in $L^{2}\left(\mathbb{R}^{3}\right)$, and that $K$ preserves (modulo a factor of 2 ) the symplectic form $\sigma$. Moreover, it can be shown that $K$ intertwines $D_{t}$ with the one parameter unitary group $U_{t}=e^{-i t H}$ on $L^{2}\left(\mathbb{R}^{3}\right)$. Therefore, $\left(K, L^{2}\left(\mathbb{R}^{3}\right), U_{t}\right)$ is a one-particle structure over $\left(S, \sigma, D_{t}\right)$.

Since $\left(\iota, \mathcal{H}_{\mu}, D_{t}\right)$ and $\left(K, L^{2}\left(\mathbb{R}^{3}\right), U_{t}\right)$ are one-particle structures over $\left(S, \sigma, D_{t}\right)$, it follows that $\left(K \circ \iota^{-1}\right)=K$ extends uniquely to a unitary operator $V$ from $\mathcal{H}_{\mu}$ onto $L^{2}\left(\mathbb{R}^{3}\right)$ :

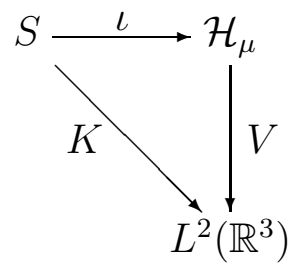

Thus, the one-particle spaces $\left(\mathcal{H}_{\mu}, D_{t}\right)$ and $\left(L^{2}\left(\mathbb{R}^{3}\right), U_{t}\right)$ are mathematically, and hence physically, equivalent. On the other hand, the two spaces certainly suggest different notions of localization. 


\subsection{Second quantization}

Once we have a one-particle space $\left(\mathcal{H}, U_{t}\right)$ in hand, the movement to a quantum field theory (i.e., "second quantization") is mathematically straightforward and uniquely determined.f In particular, let $\mathcal{F}(\mathcal{H})$ denote the "Fock space" over $\mathcal{H}$. That is,

$$
\mathcal{F}(\mathcal{H})=\mathbb{C} \oplus \mathcal{H} \oplus \mathcal{H}^{2} \oplus \mathcal{H}^{3} \oplus \cdots,
$$

where $\mathcal{H}^{n}$ is the $n$-fold symmetric tensor product of $\mathcal{H}$. As usual we let

$$
\Omega=1 \oplus 0 \oplus 0 \oplus \cdots
$$

denote the vacuum vector in $\mathcal{F}(\mathcal{H})$. For each $f \in \mathcal{H}$, we define the creation $a^{+}(f)$ and annihilation $a(f)$ operators on $\mathcal{F}(\mathcal{H})$ as usual, and we let $\Phi(f)$ denote the self-adjoint closure of the unbounded operator

$$
2^{-1 / 2}\left(a(f)+a^{+}(f)\right) .
$$

If we let $W(f)=\exp \{i \Phi(f)\}$, then the $W(f)$ satisfy the Weyl form of the canonical commutation relations:

$$
W(f) W(g)=e^{-i \operatorname{Im}(f, g) / 2} W(f+g),
$$

and vacuum expectation values are given explicitly by

$$
\langle\Omega, W(f) \Omega\rangle=\exp \left(-\|f\|^{2} / 4\right) .
$$

The dynamical group on $\mathcal{F}(\mathcal{H})$ is given by the "second quantization" $\Gamma\left(U_{t}\right)=$ $e^{i t d \Gamma(H)}$ of the dynamical group $U_{t}=e^{i t H}$ on $\mathcal{H}$, and the vacuum vector $\Omega$ is the unique eigenvector of the Hamiltonian $d \Gamma(H)$ with eigenvalue 0.

\section{Local algebras and the Reeh-Schlieder the- orem}

To this point we have only discussed the global structure of the free Bose field model. The physical observables for the free Bose field are given by the self-adjoint operators on Fock space $\mathcal{F}(\mathcal{H})$. We equip this model with

\footnotetext{
${ }^{4}$ For a more detailed exposition, see Bratteli and Robinson 1997, Section 5.2.
} 
a local structure when we define a correspondence between regions in space and "subalgebras" of observables. This labelling may be done for various purposes, but the traditional motivation was to indicate those observables that can (in theory) be measured in that region of space.

Now, each real-linear subspace $E$ of the one-particle space $\mathcal{H}$ gives rise naturally to a subalgebra of operators, viz., the algebra generated by the Weyl operators $\{W(f): f \in E\}$. Thus, a localization scheme needs only to determine which real-linear subspace of $\mathcal{H}$ should be taken as corresponding to a region $G$ in physical space. It is on this point that the Newton-Wigner localization scheme disagrees with the standard localization scheme. In the remainder of this section, I discuss the standard localization scheme and its consequences.

The standard localization scheme assigns to the spatial region $G$ the subset $S(G) \subseteq \mathcal{H}_{\mu}$ of Cauchy data localized in $G$. That is, if $C^{\infty}(G)$ denotes the subspace of $C_{0}^{\infty}\left(\mathbb{R}^{3}\right)$ of functions with support in $G$, then

$$
S(G)=C^{\infty}(G) \oplus C^{\infty}(G)
$$

is a real-linear subspace of $\mathcal{H}_{\mu}$. (Note that $S(G)$ is not closed nor, as we shall soon see, complex-linear.) Thus, in the Newton-Wigner representation, the classical localization scheme assigns $G$ to the real-linear subspace $V(S(G))$ of $L^{2}\left(\mathbb{R}^{3}\right)$. When no confusion can result, I will suppress reference to the unitary operator $V$ and simply use $S(G)$ to denote the pertinent subspace in either concrete version of the one-particle space.

Note that the correspondence $G \mapsto S(G)$ is monotone; i.e., if $G_{1} \subseteq G_{2}$ then $S\left(G_{1}\right) \subseteq S\left(G_{2}\right)$. Moreover, if $G_{1} \cap G_{2}=\emptyset$, then $S\left(G_{1}\right)$ and $S\left(G_{2}\right)$ are "symplectically orthogonal." That is, if $f \in S\left(G_{1}\right)$ and $g \in S\left(G_{2}\right)$, then $\operatorname{Im}(f, g)=0$. Indeed, if $u_{0} \oplus u_{1} \in S\left(G_{1}\right)$ and $v_{0} \oplus v_{1} \in S\left(G_{2}\right)$, then

$$
\sigma\left(u_{0} \oplus u_{1}, v_{0} \oplus v_{1}\right)=\int_{\mathbb{R}^{3}}\left(u_{0} v_{1}-u_{1} v_{0}\right) d^{3} \mathbf{x}=0
$$

since the $u_{i}$ and $v_{i}$ have disjoint regions of support.

Now, we say that a Weyl operator $W(f)$ acting on $\mathcal{F}(\mathcal{H})$ is classically localized in $G$ just in case $f \in S(G)$. ("Classically" here refers simply to the fact that our notion of localization is derived from the local structure of the classical phase space $S$.) Let $\mathbf{B}(\mathcal{F}(\mathcal{H}))$ denote the algebra of bounded operators on $\mathcal{F}(\mathcal{H})$. We then define the subalgebra $\mathfrak{R}(G) \subseteq \mathbf{B}(\mathcal{F}(\mathcal{H}))$ of operators classically localized in $G$ to be the "von Neumann algebra" generated by the 
Weyl operators classically localized in $G$. That is, $\mathfrak{R}(G)$ consists of arbitrary linear combinations and "weak limits" of Weyl operators classically localized in $G$.

If $\mathfrak{R} \subseteq \mathbf{B}(\mathcal{F}(\mathcal{H}))$, we let $\mathfrak{R}^{\prime}$ denote all operators in $\mathbf{B}(\mathcal{F}(\mathcal{H}))$ that commute with every operator in $\mathfrak{R}$. If $\mathfrak{R}$ contains $I$ and is closed under taking adjoints, then von Neumann's "double commutant theorem" entails that $\left(\mathfrak{R}^{\prime}\right)^{\prime}$ is the von Neumann algebra generated by $\mathfrak{R}$. Thus, we have

$$
\mathfrak{R}(G)=\{W(f): f \in S(G)\}^{\prime \prime}
$$

In order also to associate unbounded operators with local regions, we say that an unbounded operator $A$ is affiliated with the local algebra $\mathfrak{R}(G)$ just in case $U^{-1} A U=A$ for any unitary operator $U \in \mathfrak{R}(G)^{\prime}$. It then follows that $\Phi(f)$ is affiliated with $\mathfrak{R}(G)$ just in case $W(f) \in \mathfrak{R}(G)$.

The correspondence $G \mapsto \mathfrak{R}(G)$ clearly satisfies isotony. That is, if $G_{1} \subseteq$ $G_{2}$ then $\mathfrak{R}\left(G_{1}\right) \subseteq \mathfrak{R}\left(G_{2}\right)$. Moreover, the local algebras also satisfy fixed-time microcausality. That is, if $G_{1} \cap G_{2}=\emptyset$ then all operators in $\mathfrak{R}\left(G_{1}\right)$ commute with all operators in $\mathfrak{R}\left(G_{2}\right)$. (This follows directly from Eq. (14) and the fact that $S\left(G_{1}\right)$ and $S\left(G_{2}\right)$ are symplectically orthogonal.)

\subsection{Anti-locality and the Reeh-Schlieder theorem}

Let $\mathfrak{R}$ be some subalgebra of $\mathbf{B}(\mathcal{F}(\mathcal{H}))$. We say that a vector $\psi \in \mathcal{F}(\mathcal{H})$ is cyclic for $\mathfrak{R}$ just in case $[\mathfrak{R} \psi]=\mathcal{F}(\mathcal{H})$, where $[\mathfrak{R} \psi]$ denotes the closed linear span of $\{A \psi: A \in \mathfrak{R}\}$. Of course, every vector in $\mathcal{F}(\mathcal{H})$, including the vacuum vector $\Omega$, is cyclic for the global algebra $\mathbf{B}(\mathcal{F}(\mathcal{H}))$ of all bounded operators on $\mathcal{F}(\mathcal{H})$. The Reeh-Schlieder theorem, however, tells us that the vacuum vector $\Omega$ is cyclic for any local algebra $\mathfrak{R}(G)$.

The first version of the Reeh-Schlieder theorem I will present is a restricted version of the theorem - due to Segal and Goodman - applicable only to the free Bose field model. The key concept in this version of the theorem is the notion of an "anti-local" operator.

Definition. An operator $A$ on $L^{2}\left(\mathbb{R}^{3}\right)$ is said to be anti-local just in case: For any $f \in L^{2}\left(\mathbb{R}^{3}\right)$ and for any open subset $G$ of $\mathbb{R}^{3}, \operatorname{supp}(f) \cap G=\emptyset$ and $\operatorname{supp}(A f) \cap G=\emptyset$ only if $f=0$.

\footnotetext{
${ }^{5}$ Since $f \mapsto W(f)$ is weakly continuous, $\mathfrak{R}(G)$ contains $W(f)$ for all $f$ in the closure of $S(G)$.
} 
Thus, in particular, an anti-local operator maps any wavefunction with support inside a bounded region to a wavefunction with infinite "tails."

The following lemma may be the most important lemma for understanding the local structure of the free Bose field model:

Lemma (Segal and Goodman 1965). The operator $H=\left(-\nabla^{2}+m^{2}\right)^{1 / 2}$ is anti-local.

This lemma has the important consequence that for any non-empty open subset $G$ of $\mathbb{R}^{3}$, the complex-linear span of $S(G)$ is dense in $\mathcal{H}$ (cf. Segal and Goodman 1965, Corollary 1). However, for any real-linear subspace $E$ of $\mathcal{H}, \Omega$ is cyclic for the algebra generated by $\{W(f): f \in E\}$ if and only if the complex-linear span of $E$ is dense in $\mathcal{H}$ (cf. Petz 1990, Proposition 7.7). Thus, the anti-locality of $H$ entails that $\Omega$ is cyclic for every local algebra.

Reeh-Schlieder Theorem. Let $G$ be any nonempty open subset of $\mathbb{R}^{3}$. Then, $\Omega$ is cyclic for $\mathfrak{R}(G)$.

What is the significance of this cyclicity result? Segal $(1964,140)$ claims that the theorem is "striking," since it entails that

"...the entire state vector space of the field could be obtained from measurements in an arbitrarily small region of space-time!"

He then goes on to claim that the result is, "quite at variance with the spirit of relativistic causality" (143). Fleming also sees the cyclicity result as counterintuitive, apparently because it does not square well with our understanding of relativistic causality. For example (cf. Fleming 2000, 4), the Reeh-Schlieder theorem entails that for any state $\psi \in \mathcal{F}(\mathcal{H})$, and for any predetermined $\epsilon$, there is an operator $A \in \mathfrak{R}(G)$ such that $\|A \Omega-\psi\|<\epsilon$. In particular, $\psi$ may be a state that differs from the vacuum only in some region $G^{\prime}$ that is disjoint (and hence spacelike separated) from $G$. If, then, $A$ is interpreted as an "operation" that can be performed in the region $G$, it follows that operations performed in $G$ can result in arbitrary changes of the state in the region $G^{\prime}$. This, then, is taken by Fleming to show that, "the local fields allow the possibility of arbitrary space-like distant effects from arbitrary localized actions" (Fleming 2000, 20).

Fleming's use of "actions" and "effects" seems to construe a local operationrepresented by an operator $A \in \mathfrak{R}(G)$-as a purely physical disturbance of 
the system; i.e., the operation here is a cause with an effect at spacelike separation. If this were the only way to think of local operations, then I would grant that the Reeh-Schlieder theorem is counterintuitive, and indeed very contrary to the spirit of relativisitic causality. However, once one makes the crucial distinction between selective and nonselective local operations, local cyclicity does not obviously conflict with relativistic causality (cf. Clifton and Halvorson 2000, Section 2). Rather than dwell on that here, however, I will proceed to spell out some of the further "counterintuitive" consequences of the Reeh-Schlieder theorem.

1. Let $G_{1}$ and $G_{2}$ be disjoint subsets of $\mathbb{R}^{3}$. Suppose that $W(f)$ is classically localized in $G_{1}$ and $W(g)$ is classically localized in $G_{2}$. Then, $\operatorname{Im}(f, g)=0$ and therefore $W(f) W(g)=W(f+g)$. Thus,

$$
\begin{aligned}
\langle\Omega, W(f) W(g) \Omega\rangle & =\exp \left(-\|f+g\|^{2} / 4\right) \\
& =\langle\Omega, W(f) \Omega\rangle \cdot\langle\Omega, W(g) \Omega\rangle \cdot e^{-\operatorname{Re}(f, g) / 2} .
\end{aligned}
$$

However, $S\left(G_{1}\right)$ and $S\left(G_{2}\right)$ are not orthogonal relative to the real part of the inner product $(\cdot, \cdot)$. Indeed, if $f=u_{0} \oplus u_{1}$ and $g=v_{0} \oplus v_{1}$, then

$$
\begin{aligned}
\operatorname{Re}(f, g) & =\left(u_{0}, H v_{0}\right)+\left(u_{1}, H^{-1} v_{1}\right) \\
& =\int_{\mathbb{R}^{3}} u_{0}\left(H v_{0}\right) d^{3} \mathbf{x}+\int_{\mathbb{R}^{3}} u_{1}\left(H^{-1} v_{1}\right) d^{3} \mathbf{x} .
\end{aligned}
$$

But since $H$ and $H^{-1}$ are anti-local, the two integrals in (22) will not generally vanish. Therefore, the vacuum state is not a product state across $\mathfrak{R}\left(G_{1}\right)$ and $\mathfrak{R}\left(G_{2}\right)$.

It should be noted, however, that the above argument does not entail that the vacuum state is "entangled" - since it could still be a mixture of product states across $\mathfrak{R}\left(G_{1}\right)$ and $\mathfrak{R}\left(G_{2}\right)$. However, it can be shown directly from the cyclicity of the vacuum vector $\Omega$ that the vacuum state is not even a mixture of product states across $\mathfrak{R}\left(G_{1}\right)$ and $\mathfrak{R}\left(G_{2}\right)$ (Halvorson and Clifton $2000)$. Moreover, the vacuum predicts a maximal violation of Bell's inequality relative to the algebras $\mathfrak{R}(G)$ and $\mathfrak{R}\left(G^{\prime}\right)$, where $G^{\prime}=\mathbb{R}^{3} \backslash G$ (Summers and Werner 1985). (Bell correlation, however, is not entailed by cyclicity.)

2. The cyclicity of the vacuum combined with (equal-time) microcausality entails that the vacuum vector is separating for any local algebra $\mathfrak{R}(G)$, where $G^{\prime}$ has non-empty interior. That is, for any operator $A \in \mathfrak{R}(G)$, if 
$A \Omega=0$ then $A=0$. In particular, for any local event-represented by projection operator $P \in \mathfrak{R}(G)$ - the probability that event will occur in the vacuum state is nonzero. Thus, the vacuum is "seething with activity" at the local level.

Since the vacuum is entangled across $\mathfrak{R}(G)$ and $\mathfrak{R}\left(G^{\prime}\right)$, it follows that the vacuum is a mixed state when restricted to the local algebra $\mathfrak{R}(G)$. In fact, when we restrict the vacuum to $\mathfrak{R}(G)$, it is maximally mixed in the sense that the vacuum may be written as a mixture with any one of a dense set of states of $\mathfrak{R}(G)$ (Clifton and Halvorson 2000). Intuitively speaking, then, the vacuum state provides minimal information about local states of affairs. This is quite similar to the singlet state, which restricts to the maximally mixed state (1/2)I on either one-particle subsystem (cf. Redhead 1995a).

3. For any annihilation operator $a(f)$, we have $a(f) \Omega=0$. Thus, $a(f)$ cannot be affiliated with the local algebra $\mathfrak{R}(G)$. Since the family of operators affiliated with $\mathfrak{R}(G)$ is closed under taking adjoints, it also follows that no creation operators are affiliated with $\mathfrak{R}(G)$.

The concreteness of the model we are dealing with allows a more direct understanding of why, mathematically speaking, local algebras do not contain creation and annihilation operators. Inverting the relation in (13), and using the fact that $f \mapsto a^{+}(f)$ is linear and $f \mapsto a(f)$ is anti-linear, it follows that

$$
\begin{aligned}
a^{+}(f) & =2^{-1 / 2}(\Phi(f)-i \Phi(i f)), \\
a(f) & =2^{-1 / 2}(\Phi(f)+i \Phi(i f)) .
\end{aligned}
$$

Thus, an algebra generated by the operators $\{W(f): f \in E\}$, will contain the creation and annihilation operators $\left\{a^{+}(f), a(f): f \in E\right\}$ only if $E$ is a complex-linear subspace of $\mathcal{H}$. This is not the case for a local algebra $\mathfrak{R}(G)$ where $E=S(G)$ is a real-linear subspace of $\mathcal{H}$. In fact, referring to the concrete one-particle space $\mathcal{H}_{\mu}$ allows us to see clearly that $S(G)$ is not invariant under the complex structure $J$. If $u_{0} \oplus u_{1} \in S(G)$, then

$$
J\left(u_{0} \oplus u_{1}\right)=-H^{-1} u_{1} \oplus H u_{0} .
$$

But since $H$ and $H^{-1}$ are anti-local, it is not the case that $H u_{0} \in C^{\infty}(G)$ or $-H^{-1} u_{1} \in C^{\infty}(G)$. Thus, $J f \notin S(G)$ when $f \in S(G)$. What is more, since the complex span of $S(G)$ is dense in $\mathcal{H}_{\mu}$, if $S(G)$ were a complex subspace, then it would follow that $\mathfrak{R}(G)=\mathbf{B}(\mathcal{F}(\mathcal{H}))$. 
3. Number operators also annihilate the vacuum. Since the vacuum is separating for local algebras, no number operator is affiliated with any local algebra. Thus, an observer in the region $G$ cannot count the number of particles in $G$ !

How should we understand the inability of local observers to count the number of particles in their vicinity? According to Redhead (1995b), a heuristic calculation shows that the local number density operator $N_{G}$ does not commute with the density operator $N_{G^{\prime}}$ (where $G^{\prime}$ is the complement of $G)$. Thus, he claims that

"...it is usual in axiomatic formulations of quantum field theory to impose a microcausality condition on physically significant local observables, viz that the associated operators should commute at space-like separation. The conclusion of this line of argument is that number densities are not physical observables, and hence we do not have to bother about trying to interpret them." (81)

While Redhead's conclusion is correct, it is instructive to note that his reasoning cannot be reproduced in a mathematically rigorous fashion. That is, there are no local number density operators - in particular, neither $N_{G}$ nor $N_{G^{\prime}}$ exist - and so it cannot be literally true that $N_{G}$ and $N_{G^{\prime}}$ fail to commute.

In order to see this, consider first the (single wavefunction) number operator $N_{f}=a^{+}(f) a(f)$, where $f$ is "classically localized" in $G$, i.e., $f \in S(G)$. Since $f \mapsto a^{+}(f)$ is linear, and $f \mapsto a(f)$ is anti-linear, it follows that $N_{f}=N_{\left(e^{i t} f\right)}$ for all $t \in \mathbb{R}$. That is, a single wavefunction number operator $N_{f}$ is invariant under phase tranformations of $f$. However, classical localization of a wavefunction is not invariant under phase transformations. Thus, it is not possible to formulate a well-defined notion of classical localization for a single wavefunction number operator.

How, though, do we define a number density operator $N_{G}$ ? Heuristically, one sets

$$
N_{G}=\int_{G} N(\mathbf{x}) d^{3} \mathbf{x}
$$

where $N(\mathbf{x})=a^{+}(\mathbf{x}) a(\mathbf{x})$. Since, however, $N(\mathbf{x})$ is not a well-defined mathematical object, Eq. (26) is a purely formal expression. Thus, we replace $N(\mathbf{x})$ with the single wavefunction number operator $N_{f}$ and we set,

$$
N_{G}=\sum_{i} N_{f_{i}}
$$


where $f_{i}$ is a basis of the real-linear subspace $S(G)$ of $\mathcal{H}$. 9 Using the fact that $N_{f}=N_{i f}$ for each $f$, it follows then that $N_{G}=N_{[G]}$, where $N_{[G]}$ is the number operator for the closed complex-linear span $[S(G)]$ of $S(G)$ in $\mathcal{H}$; and the anti-locality of $H$ entails that $[S(G)]=\mathcal{H}$. Therefore, the operator we defined in Eq. (27) turns out to be the total number operator $N$.

4. The Reeh-Schlieder theorem also has implications for the internal structure of the local algebra $\mathfrak{R}(G)$. In particular, the local algebra $\mathfrak{R}(G)$ is what is called a "type III" von Neumann algebra (Araki 1964). (The algebra $\mathbf{B}(\mathcal{F}(\mathcal{H}))$ of all bounded operators on $\mathcal{F}(\mathcal{H})$ is called a type I von Neumann algebra.) From a physical point of view, this is significant since type III algebras contain only infinite-dimensional projections - which entails that there are strict limits on our ability to "isolate" a local system from outside influences (Clifton and Halvorson 2000). Type III algebras also have no pure (normal) states.

\section{Newton-Wigner localization}

In the previous section, we saw that the standard localization scheme $G \mapsto$ $\mathfrak{R}(G)$ has a number of "counterintuitive" features, all of which follow from the Reeh-Schlieder theorem. These counterintuitive features prompted Segal (1964) and Fleming (2000) to suggest a reworking of the correspondence between spatial regions and subalgebras of observables. In this section I give a mathematically rigorous rendering of the Segal-Fleming proposal, and I show how it avoids both the Reeh-Schlieder theorem and its consequences. (Here I deal only with Fleming's first proposal, prior to his generalization to "covariant fields.")

Recall that a localization scheme defines a correspondence between regions in space and real-linear subspaces of the one-particle space $\mathcal{H}$. The Newton-Wigner localization scheme defines this correspondence in precisely the way it is done in elementary quantum mechanics: A region $G$ in $\mathbb{R}^{3}$ corresponds to the subspace $L^{2}(G) \subseteq L^{2}\left(\mathbb{R}^{3}\right)$ of wavefunctions with probability amplitude vanishing (almost everywhere) outside of $G$. We may then use the unitary mapping $V$ between $\mathcal{H}_{\mu}$ and $L^{2}\left(\mathbb{R}^{3}\right)$ to identify the subspace

\footnotetext{
${ }^{6}$ Actually, this infinite sum is also a formal expression, since it sums unbounded operators. A technically correct definition would define $N_{G}$ as an upper bound of quadratic forms (see Bratteli and Robinson 1997).
} 
$V^{-1} L^{2}(G)$ of Newton-Wigner localized wavefunctions in $\mathcal{H}_{\mu}$. Hereafter, I will suppress reference to $V^{-1}$ and use $L^{2}(G)$ to denote the pertinent subspace in either concrete version of the one-particle space.

Note that the correspondence $G \mapsto L^{2}(G)$ is monotone; i.e., if $G_{1} \subseteq G_{2}$ then $L^{2}\left(G_{1}\right) \subseteq L^{2}\left(G_{2}\right)$. Moreover, if $G_{1} \cap G_{2}=\emptyset$, then $L^{2}\left(G_{1}\right)$ and $L^{2}\left(G_{2}\right)$ are fully orthogonal - a key difference between NW localization and classical localization.

Now, we say that a Weyl operator $W(f)$ acting on $\mathcal{F}(\mathcal{H})$ is $N W$-localized in $G$ just in case $f \in L^{2}(G)$. We then define the algebra $\mathfrak{R}_{N W}(G)$ of NWlocalized operators on $\mathcal{F}(\mathcal{H})$ as the von Neumann algebra generated by the Weyl operators NW-localized in $G$. That is,

$$
\mathfrak{R}_{N W}(G)=\left\{W(f): f \in L^{2}(G)\right\}^{\prime \prime} .
$$

Clearly, the correspondence $G \mapsto \mathfrak{R}_{N W}(G)$ satisfies isotony. Moreover, since $G_{1} \cap G_{2}=\emptyset$ entails that $L^{2}\left(G_{1}\right)$ and $L^{2}\left(G_{2}\right)$ are orthogonal subspaces of $\mathcal{H}$, the correspondence $G \mapsto \mathfrak{R}_{N W}(G)$ satisfies fixed-time microcausality. Thus, at least in this fixed-time formulation, the NW localization scheme appears to have all the advantages of the classical localization scheme. I will now proceed to spell out some features of the NW localization scheme that may make it seem more attractive than the standard localization scheme.

If $G$ is an open subset of $\mathbb{R}^{3}$, then

$$
L^{2}\left(\mathbb{R}^{3}\right)=L^{2}\left(G \cup G^{\prime}\right)=L^{2}(G) \oplus L^{2}\left(G^{\prime}\right) .
$$

Accordingly, if we let $\mathcal{F}_{G}=\mathcal{F}\left(L^{2}(G)\right)$ and $\mathcal{F}_{G^{\prime}}=\mathcal{F}\left(L^{2}\left(G^{\prime}\right)\right)$ then it follows that

$$
\mathcal{F}(\mathcal{H})=\mathcal{F}_{G} \otimes \mathcal{F}_{G^{\prime}}
$$

(Here the equality sign is intended to denote that there is a natural isomorphism between $\mathcal{F}(\mathcal{H})$ and $\mathcal{F}_{G} \otimes \mathcal{F}_{G^{\prime}}$.) Moreover, the vacuum vector $\Omega \in \mathcal{F}(\mathcal{H})$ is the product $\Omega_{G} \otimes \Omega_{G^{\prime}}$ of the respective vacuum vectors in $\mathcal{F}_{G}$ and $\mathcal{F}_{G^{\prime}}$. By definition, $\Phi(f)$ is affiliated with $\mathfrak{R}_{N W}(G)$ when $f \in L^{2}(G)$. Since $L^{2}(G)$ is a complex-linear subspace of $\mathcal{H}$, it follows that $\Phi(i f)$ is also affiliated with $\mathfrak{R}_{N W}(G)$, and hence that $a^{+}(f), a(f)$, and $N_{f}$ are all affiliated with $\mathfrak{R}_{N W}(G)$. If we let $U$ denote the unitary operator that maps $\mathcal{F}_{G} \otimes \mathcal{F}_{G^{\prime}}$ naturally onto $\mathcal{F}(\mathcal{H})$, then it is not difficult to see that

$$
U^{-1} a^{+}(f) U=a_{G}^{+}(f) \otimes I,
$$


where $a_{G}^{+}(f)$ is the creation operator on $\mathcal{F}_{G}$. Thus, we also have $U^{-1} a(f) U=$ $a_{G}(f) \otimes I$, and since the creation and annihilation operators $\left\{a_{G}^{ \pm}(f): f \in\right.$ $\left.L^{2}(G)\right\}$ form an irreducible set of operators on $\mathcal{F}_{G}$, it follows that

$$
\begin{aligned}
\mathfrak{R}_{N W}(G) & =\mathbf{B}\left(\mathcal{F}_{G}\right) \otimes I \\
\mathfrak{R}_{N W}\left(G^{\prime}\right) & =I \otimes \mathbf{B}\left(\mathcal{F}_{G^{\prime}}\right) .
\end{aligned}
$$

(Again, equality here means there is a natural isomorphism.)

It follows then that acting on $\Omega=\Omega_{G} \otimes \Omega_{G^{\prime}}$ with elements from $\mathfrak{R}_{N W}(G)$ results only in vectors of the form $\psi \otimes \Omega_{G^{\prime}}$ for some $\psi \in \mathcal{F}_{G}$. Thus, the vacuum is not cyclic for the local algebra $\mathfrak{R}_{N W}(G)$.

1. It is obvious from the preceding that the vacuum is a product state across $\mathfrak{R}_{N W}(G)$ and its complement $\mathfrak{R}_{N W}\left(G^{\prime}\right)$. This also follows directly from the fact that $L^{2}(G)$ and $L^{2}\left(G^{\prime}\right)$ are fully orthogonal subspaces of $\mathcal{H}$. Indeed, let $W(f) \in \mathfrak{R}_{N W}(G)$ and $W(g) \in \mathfrak{R}_{N W}\left(G^{\prime}\right)$. Then since $\|f+g\|^{2}=$ $\|f\|^{2}+\|g\|^{2}$, it follows that

$$
\begin{aligned}
\langle\Omega, W(f) W(g) \Omega\rangle & =\langle\Omega, W(f+g) \Omega\rangle \\
& =\exp \left(-\|f+g\|^{2} / 4\right) \\
& =\langle\Omega, W(f) \Omega\rangle \cdot\langle\Omega, W(g) \Omega\rangle .
\end{aligned}
$$

2. Restricting the vacuum state $\Omega$ to $\mathfrak{R}_{N W}(G)$ is equivalent to restricting the product state $\Omega_{G} \otimes \Omega_{G}$, to $\mathbf{B}\left(\mathcal{F}_{G}\right) \otimes I$. Thus, the restriction of $\Omega$ to $\mathfrak{R}_{N W}(G)$ is pure, and the global vacuum provides a "maximally specific" description of local states of affairs.

3. If $\left\{f_{i}\right\}$ is an orthonormal basis of $L^{2}(G)$, then the number operator $N_{G}=\sum_{i} N_{f_{i}}$ is affiliated with $\mathfrak{R}_{N W}(G)$. Moreover, the number operator $N_{G^{\prime}}$ is affiliated with $\mathfrak{R}_{N W}\left(G^{\prime}\right)$, and by microcausality we have $\left[N_{G}, N_{G^{\prime}}\right]=$ 0 . We may also see this by employing the correspondence between $\mathcal{F}(\mathcal{H})$ and $\mathcal{F}_{G} \otimes \mathcal{F}_{G^{\prime}}$. The Fock space $\mathcal{F}_{G}$ has its own total number operator $\widetilde{N}_{G}$. Similarly, $\mathcal{F}_{G^{\prime}}$ has its own total number operator $\widetilde{N}_{G^{\prime}}$. Obviously then, $\widetilde{N}_{G} \otimes I$ is affiliated with $\mathbf{B}\left(\mathcal{F}_{G}\right) \otimes I$, and $I \otimes \widetilde{N}_{G^{\prime}}$ is affiliated with $I \otimes \mathbf{B}\left(\mathcal{F}_{G^{\prime}}\right)$. Just as obviously, $\widetilde{N}_{G} \otimes I$ commutes with $I \otimes \widetilde{N}_{G^{\prime}}$.

4. As can be seen from Eq. (32), the local algebra $\mathfrak{R}_{N W}(G)$ is a type I von Neumann algebra. According to Segal $(1964,140)$, this is precisely the 
structure of local algebras that is "suggested by considerations of causality and empirical accessibility."

\section{The full strength of Reeh-Schlieder}

The results of the previous two sections speak for themselves: The NewtonWigner localization scheme results in a mathematical structure that appears to be much more in accord with our a priori physical intuitions than the structure obtained from the standard localization scheme. In this section, however, I show that the NW localization scheme "avoids" the Reeh-Schlieder theorem in only a trivial sense, and I show that the NW localization scheme has its own counterintuitive features without parallel in the standard localization scheme.

First, while the NW-local algebras avoid cyclicity of the vacuum vector, they still have a dense set of cyclic vectors.

Theorem 1. $\mathfrak{R}_{N W}(G)$ has a dense set of cyclic vectors in $\mathcal{F}(\mathcal{H})$.

Proof. Since the Hilbert spaces $\mathcal{F}_{G}$ and $\mathcal{F}_{G^{\prime}}$ have the same (infinite) dimension, it follows from Theorem 4 of (Clifton et al. 1998) that $\mathfrak{R}_{N W}(G)=$ $\mathbf{B}\left(\mathcal{F}_{G}\right) \otimes I$ has a dense set of cyclic vectors in $\mathcal{F}(\mathcal{H})=\mathcal{F}_{G} \otimes \mathcal{F}_{G^{\prime}}$.

Thus, if the worry about the Reeh-Schlieder theorem is about cyclicity in general, adopting the NW localization scheme does nothing to alleviate this worry.

Perhaps, however, the worry about the Reeh-Schlieder theorem is specifically a worry about cyclicity of the vacuum state. (One wonders, though, why this would be worse than cyclicity of any other state.) Even so, I argue now that the NW localization scheme does not avoid the "vacuum-specific" consequences of the full Reeh-Schlieder theorem.

Let $\mathcal{K}$ be an arbitrary Hilbert space, representing the state space of some quantum field theory. (For example, $\mathcal{K}=\mathcal{F}(\mathcal{H})$ in the case of the free Bose field.) Suppose also that there is a representation $\mathbf{a} \mapsto U(\mathbf{a})$ of the spacetime translation group in the group of unitary operators on $\mathcal{K}$. Given such a representation, there is a "four operator" $\mathbf{P}$ on $\mathcal{K}$ such that $U(\mathbf{a})=e^{i \mathbf{a} \cdot \mathbf{P}}$.

\footnotetext{
${ }^{7}$ Cf. Fleming's claim that, "...it is remarkable that any state can have enough structure within an arbitrarily small region, $O$, to enable even the mathematical reconstituting of essentially the whole state space" (Fleming 2000, 5).
} 
We say that the representation $\mathbf{a} \mapsto U(\mathbf{a})$ satisfies the spectrum condition just in case the spectrum of $\mathbf{P}$ is contained in the forward light cone. From a physical point of view, the spectrum condition corresponds to the assumption that (a) all physical effects propagate at velocities at most the speed of light, and (b) energy is positive. Note, consequently, that the spectrum condition is a purely global condition, and so is not likely to be a source of dispute between proponents of differing localization schemes.

A net of local observable algebras is an assigment $O \mapsto \mathfrak{A}(O)$ of open regions in Minkowski spacetime to von Neumann subalgebras of $\mathbf{B}(\mathcal{K})$. (Note that this definition is not immediately pertinent to the localization schemes presented in Sections 3 and 4, since they gave an assignment of algebras to open regions in space at a fixed time.) The full Reeh-Schlieder theorem will apply to this net if it satisfies the following postulates:

1. Isotony: If $O_{1} \subseteq O_{2}$, then $\mathfrak{A}\left(O_{1}\right) \subseteq \mathfrak{A}\left(O_{2}\right)$.

2. Translation Covariance: $U(\mathbf{a})^{-1} \mathfrak{A}(O) U(\mathbf{a})=\mathfrak{A}(O+\mathbf{a})$.

3. Weak Additivity: For any open $O \subseteq M$, the set

$$
\bigcup_{\mathbf{a} \in M} U(\mathbf{a})^{-1} \mathfrak{A}(O) U(\mathbf{a})
$$

of operators is irreducible (i.e., leaves no subspace of $\mathcal{K}$ invariant).

In this general setting, a vacuum vector $\Omega$ can be taken to be any vector invariant under all spacetime translations $U(\mathbf{a})$.

Full Reeh-Schlieder Theorem. Suppose that $\{\mathfrak{A}(O)\}$ is a net of local observable algebras satisfying postulates 1-3. Then, for any open region $O$ in Minkowski spacetime, $\Omega$ is cyclic for $\mathfrak{A}(O)$.

Note that the Reeh-Schlieder theorem does not require the postulate of microcausality (i.e., if $A \in \mathfrak{A}\left(O_{1}\right)$ and $B \in \mathfrak{A}\left(O_{2}\right)$, where $O_{1}$ and $O_{2}$ are spacelike separated, then $[A, B]=0)$. \

For the standard localization scheme, there is a straightforward connection between the full Reeh-Schlieder theorem and the fixed-time version given in Section 3. In particular, there is an alternative method for describing the

\footnotetext{
${ }^{8}$ To see that microcausality is logically independent from postulates $1-3$, take the trivial localization scheme: $\mathfrak{A}(O)=\mathbf{B}(\mathcal{K})$, for each $O$.
} 
standard localization scheme that involves appeal to spacetime regions rather than space regions at a fixed time (see Horuzhy 1988, Chapter 4). It then follows that $\mathfrak{R}(G)=\mathfrak{A}\left(O_{G}\right)$, where $O_{G}$ is the "domain of dependence" of the spatial region $G$. Thus, the fixed-time version of the Reeh-Schlieder theorem may be thought of as corollary of the full Reeh-Schlieder theorem in connection with the fact that $\mathfrak{R}(G)=\mathfrak{A}\left(O_{G}\right)$.

Segal and Fleming avoid the fully general version of the Reeh-Schlieder theorem only by remaining silent about how we ought to assign algebras of observables to open regions of spacetime. I Since, however, the typical quantum field theory cannot be expected to admit a fixed-time $(3+1)$ formulation (cf. Haag 1992, 59), it is not at all clear that they have truly avoided the Reeh-Schlieder theorem in any interesting sense. It would certainly be interesting to see which, if any, of the full Reeh-Schlieder theorem's three premises would be rejected by a more general NW localization scheme.

However, we need not speculate about the possibility that the full ReehSchlieder theorem will apply to some generalization of NW localization scheme: The Reeh-Schlieder theorem already has counterintuitive consequences for the fixed-time NW localization scheme. In particular, although the vacuum $\Omega$ is not cyclic under operations NW-localized in some spatial region $G$ at a single time, $\Omega$ is cyclic under operators NW-localized in $G$ within an arbitrary short time interval. Before I give the precise version of this result, I should clarify some matters concerning the relationship between the dynamics of the field and local algebras.

In the standard localization scheme, the dynamics of local algebras may be thought of two ways. On the one hand, we may think of the assignment $G \mapsto \mathfrak{R}(G)$ as telling us, once and for all, which observables are associated with the region $G$, in which case the state of $\mathfrak{R}(G)$ (i.e., the reduced state of the entire field) changes via the unitary evolution $U(t)$ (Schrödinger picture). On the other hand, we may think of the state of the field as fixed, in which case the algebra $\mathfrak{R}(G)$ evolves over time to the algebra $U(t)^{-1} \mathfrak{R}(G) U(t)$ (Heisenberg picture). Thus, $U(t)^{-1} \mathfrak{R}(G) U(t)$ gives those operators classically localized in $G$ at time $t$. The Schrödinger picture is particularly intuitive in this case, since it mimics the dynamics of a classical field where quantities associated with points in space change their values over time.

\footnotetext{
${ }^{9}$ It is essential for the proof of the full Reeh-Schlieder theorem that the region $O$ has some "temporal extension": The theorem uses the fact that if $A \in \mathfrak{A}\left(O_{1}\right)$ where $O_{1} \subset O$, then $U(\mathbf{a})^{-1} A U(\mathbf{a}) \in \mathfrak{A}(O)$ for sufficiently small $\mathbf{a}$ in four independent directions.
} 
Now, neither Segal nor Fleming explain how we should think of the dynamics of the NW-local algebras. Presumably, however, we are to think of the dynamics of the NW-local algebras in precisely the same way as we think of the dynamics of the standard local algebras. UI In particular, we may suppose that the state of the field is, at all times, the vacuum state $\Omega$, and that $U(t)^{-1} \mathfrak{R}_{N W}(G) U(t)$ gives those operators NW-localized in $G$ at time $t$.

Now for any $\Delta \subseteq \mathbb{R}$ let

$$
\mathbf{S}_{\Delta}=\left\{U(t)^{-1} A U(t): A \in \mathfrak{R}_{N W}(G), t \in \Delta\right\} .
$$

That is, $\mathbf{S}_{\Delta}$ consists of those operators NW-localized in $G$ at some time $t \in \Delta$.

Theorem 2. For any interval $(a, b)$ around $0, \Omega$ is cyclic for $\mathbf{S}_{(a, b)}$.

Sketch of proof: Let $\left[\mathbf{S}_{(a, b)} \Omega\right]$ denote the closed linear span of $\{A \Omega: A \in$ $\left.\mathbf{S}_{(a, b)}\right\}$. Since the infinitesimal generator $d \Gamma(H)$ of the group $U(t)$ is positive, Kadison's "little Reeh-Schlieder theorem" (1970) entails that $\left[\mathbf{S}_{(a, b)} \Omega\right]=$ $\left[\mathbf{S}_{\mathbb{R}} \Omega\right]$. However, $\left[\mathbf{S}_{\mathbb{R}} \Omega\right]=\mathcal{F}(\mathcal{H})$; i.e., $\Omega$ is cyclic under operators NWlocalized in $G$ over all times (Segal 1964, 143). Therefore, $\Omega$ is cyclic for $\mathbf{S}_{(a, b)}$.

In Fleming's language, then, the NW-local fields "allow the possibility of arbitrary space-like distant effects" from actions localized in an arbitrarily small region of space over an arbitrarily short period of time. Is this any less "counterintuitive" than the instantaneous version of the Reeh-Schlieder theorem for the standard localization scheme?"

Finally, we are in a position to see explicitly a "counterintuitive" feature of the NW localization scheme that is not shared by the standard localization scheme: NW-local operators fail to commute at spacelike separation. For this, choose mutually disjoint regions $G_{1}$ and $G_{2}$ in $\mathbb{R}^{3}$, and choose an interval $(a, b)$ around 0 so that $O_{1}:=\cup_{t \in(a, b)}\left(G_{1}+t\right)$ and $O_{2}:=\cup_{t \in(a, b)}\left(G_{2}+t\right)$ are

\footnotetext{
${ }^{10}$ It is conceivable that Segal or Fleming have some different idea concerning the relationship between NW-local algebras at different times. For example, perhaps even in the Schrödinger picture, the map $G \mapsto \mathfrak{R}_{N W}(G)$ should be thought of as time-dependent. Although this is surely a formal possibility, it is exceedingly difficult to understand what it might mean, physically, to have a time-dependent association of physical magnitudes with regions in space.

${ }^{11}$ One may, however, reject the interpretation of elements of $\mathfrak{R}_{N W}(G)$ as operations that can be performed in $G$. I return to this point in the next section.
} 
spacelike separated. Let $\mathfrak{A}_{N W}\left(O_{i}\right)$ be the von Neumann algebra generated by

$$
\bigcup_{t \in(a, b)} U(t)^{-1} \mathfrak{R}_{N W}\left(G_{i}\right) U(t) .
$$

Then it follows from Theorem 2 that the vacuum is cyclic for $\mathfrak{A}_{N W}\left(O_{2}\right)$. However, since $\mathfrak{A}_{N W}\left(O_{1}\right) \supseteq \mathfrak{R}_{N W}(G)$ contains annihilation operators and number operators, it follows that $\mathfrak{A}_{N W}\left(O_{1}\right)$ and $\mathfrak{A}_{N W}\left(O_{2}\right)$ do not satisfy microcausality. (Microcausality, in conjunction with cyclicity of the vacuum vector, would entail that the vacuum vector is separating.) More specifically, while the algebras $U(t)^{-1} \mathfrak{R}_{N W}\left(G_{1}\right) U(t)$ and $U(t)^{-1} \mathfrak{R}_{N W}\left(G_{2}\right) U(t)$ do satisfy microcausality for any fixed $t$, microcausality does not generally hold for the algebras $U(t)^{-1} \mathfrak{R}_{N W}\left(G_{1}\right) U(t)$ and $U(s)^{-1} \mathfrak{R}_{N W}\left(G_{2}\right) U(s)$ when $t \neq s$ (despite the fact that $G_{1}+t$ and $G_{2}+s$ are spacelike separated).

It would be naive at this stage to claim that failure of generalized microcausality provides a simple reductio on the NW-localization scheme. As I will argue in the next section, however, the failure of generalized microcausality for the NW-local algebras leaves little room for making any physical sense of the NW localization scheme.

\section{$6 \quad$ Local properties and local measurements}

The assignment $G \mapsto \mathfrak{R}(G)$ was originally taken to have the operational meaning that $\mathfrak{R}(G)$ consists of those observables that are measurable in the region $G$. What is the intended meaning of the alternative assignment $G \mapsto \mathfrak{R}_{N W}(G)$ ? Segal $(1964,142)$ presents the NW localization scheme as a contrasting claim about what can be measured in the spatial region $G$ :

"From an operational viewpoint it is these variables [i.e., $\Phi(f)$ with $\left.f \in L^{2}(G)\right]$...that appear as the localized field variables, and the ring $\mathfrak{R}_{N W}(G)$...appears as the appropriate ring of local field observables, rather than the ring $\mathfrak{R}(G) \ldots$ (notation adapted)

According to this interpretation, the standard localization scheme and the NW localization scheme present us with two empirically inequivalent versions of quantum field theory. (For example, the vacuum displays Bell correlations relative to the algebras $\mathfrak{R}(G)$ and $\mathfrak{R}\left(G^{\prime}\right)$, while the vacuum is a product state across $\mathfrak{R}_{N W}(G)$ and $\mathfrak{R}_{N W}\left(G^{\prime}\right)$.) Thus, deciding which localization scheme is "correct" would be a matter of experiment, not a matter of interpretation. 
There is, however, also a conceptual difficulty with interpreting $\mathfrak{R}_{N W}(G)$ as the algebra of observables measurable in $G$. In particular, if $[A, B] \neq 0$, then measurement of $A$ can affect the statistics for outcomes of $B$ and vice versa. Thus, if $\mathfrak{R}_{N W}(G)$ is what is measurable in $G$, then the failure of generalized microcausality for the NW-local algebras would pose the threat of causal anomalies.

This difficulty posed by the failure of generalized microcausality is clear to Fleming. In response, he and Butterfield $(1999,158)$ note that

"...one naturally assumes that one can interpret the association of an operator with a spacetime region as implying that one can measure it by performing operations confined to that region,"

and they assert that they, "...question [this] interpretive assumption" (159). But if not local measurability, what does association of an observable with a spatial region mean? Fleming $(2000,21)$ claims that NW position operators,

"...are more closely related than the local field coordinate to assessments of where, on hyperplanes and in space-time, objects, systems, their localizable properties and phenomena are located."

It seems then that Fleming intends something along the lines of:

$(\dagger)$ The projections in $\mathfrak{R}_{N W}(G)$ correspond to the properties of the system that are localized in $G$.

But what does Fleming mean by saying that a property is localized in a spatial region $G$ ? And why would the properties localized in $G$ differ from what can be measured in $G$ ?

Although Fleming has not offered a "philosophical account" of localized properties, he has provided analogies from classical mechanics in order to prime our intuitions about physical quantities that may "pertain to" a region, without being measurable in that region (cf. Fleming 2000; Fleming and Butterfield 1999). For example, take the center of mass $C$ of a spatially extended system. At a given time, $C$ is located at a point $\mathbf{x}$ in space, but $C$ is not measurable at $\mathbf{x}$ or even in spatial regions immediately surrounding $\mathbf{x}$. Perhaps then we can think of NW-localized quantities as similar to center of mass, center of charge, and their ilk.

This analogy, however, conceals an equivocation in the meaning of "localized." To see this clearly, let me distinguish two types of localized quantities 
in classical mechanics. On the one hand, a physical quantity $Q$ may be permanently attached to some point $\mathbf{x}$ in space, in which case the values of $Q$ are given by vectors (or more generally, tensors) in the tangent space $T_{\mathbf{x}}$ over $\mathbf{x}$ (e.g., magnetic field strength at $\mathbf{x}$ ). I will refer to this first type of localized quantity as fixedly-localized. On the other hand, some quantities take vectors in physical space as their values (e.g., center of mass of a spatially extended system, or position of a point particle). I will refer to this latter type of localized quantity as variably-localized.

I grant that there is a sense in which v-localized quantities are "localized," despite the fact that they may not be locally measurable (e.g., center of mass). However, analogies to v-localized quantities go no distance in clarifying the localization map $G \mapsto \mathfrak{R}_{N W}(G)$, since this is a permanent assignment (flocalization) of physical quantities to regions in space. What we really need, then, is an example of a f-localized quantity that is not locally measurable.

One might claim that examples of such quantities are readily forthcoming: Let $C$ be center of mass, and let $Q$ be the quantity that assumes the value 1 if $C=\mathbf{x}$, and 0 if $C \neq \mathbf{x}$. Obviously, $Q$ will not typically be measurable in the vicinity of $\mathbf{x}$. But shouldn't we say that $Q$ always "pertains to" $\mathbf{x}$, or is f-localized at $\mathbf{x}$ ?

The intuition behind thinking that $Q$ "pertains to" $\mathbf{x}$ seems to be based on the fact that $Q$ tells us something about $\mathbf{x}$, viz., whether it is $C$ 's value. However, if this a sufficient condition for $Q$ 's pertaining to $\mathbf{x}$, then $Q$ pertains to every point in space. Indeed, let $\mathbf{y}$ be another vector in $\mathbb{R}^{3}$ and introduce the new quantity $C^{\prime}=C+(\mathbf{y}-\mathbf{x})$. Then $Q=1$ if and only if $C^{\prime}=\mathbf{y}$, and so the previous line of reasoning would imply that $Q$ pertains to y (since $Q$ tells us whether $\mathbf{y}$ is the value of $C^{\prime}$ ). What we should conclude, then, is that $Q$ is not f-localized at $\mathbf{x}$ in the same sense that a field quantity may be localized at $\mathbf{x}$. Thus, we have yet to find an example of a f-localized quantity that is not locally measurable.

In summary, while it is clear what it means for a physical quantity to be v-localized in $G$ but not measurable in $G$, it is by no means clear what it would mean for a physical quantity to be f-localized in $G$ but not measurable in $G$. As a result, it is also not clear how we should interpret the localization $\operatorname{map} G \mapsto \mathfrak{R}_{N W}(G)$. 


\section{Conclusion}

Introduction of the NW localization scheme into quantum field theory was an ingenious move. By means of one deft transformation, it appears to thwart the Reeh-Schlieder theorem and to restore the "intuitive" picture of localization from non-relativistic quantum mechanics. However, there are many reasons to doubt that Newton-Wigner has truly spared us of the counterintuitive consequences of the Reeh-Schlieder theorem. First, NW-local algebras still have a dense set of cyclic vectors. Second, since general quantum field theories cannnot be expected to admit a fixed-time formulation, it is not clear that the NW localization scheme has any interesting level of generality. Third, NW-local operations on the vacuum over an arbitrarily short period of time do generate the state space of the entire field. And, finally, the failure of generalized microcausality for the NW localization scheme leaves us without any natural physical interpretation of the correspondence $G \mapsto \mathfrak{R}_{N W}(G)$.

After showing that the Reeh-Schlieder theorem fails for NW-local algebras, Fleming $(2000,11)$ states that, "Now it is clear why it would be worthwhile to see the NW fields as covariant structures." While there may be very good reasons for seeing the NW fields as covariant structures, avoiding the Reeh-Schlieder theorem is not one of them.

Acknowledgments: I am grateful for helpful correspondence from Jeremy Butterfield and Bernard Kay, and I am extremely grateful to Rob Clifton for his input throughout this project.

\section{References:}

Araki, Huzihiro (1964), "Types of von Neumann algebras of local observables for the free scalar field", Progress of Theoretical Physics 32: 956-961.

Bratteli, Ola, and Derek Robinson (1997), Operator Algebras and Quantum Statistical Mechanics, Vol. 2. NY: Springer.

Clifton, Rob, David Feldman, Hans Halvorson, Michael Redhead, and Alex Wilce (1998), "Superentangled states", Physical Review A 58: 135-145.

Clifton, Rob, and Hans Halvorson (2000), "Entanglement and open systems in algebraic quantum field theory", Studies in the History and Philosophy of Modern Physics, forthcoming.

Fleming, Gordon (1996), "Just how radical is hyperplane dependence?", in Rob Clifton (ed.), Perspectives on Quantum Reality. Dordrecht: Kluwer.

Fleming, Gordon (2000), "Reeh-Schlieder meets Newton-Wigner", in PSA 
1998, v. 2, forthcoming.

Fleming, Gordon, and Jeremy Butterfield (1999), "Strange positions", in Jeremy Butterfield and Constantine Pagonis (eds.), From Physics to Philosophy, NY: Cambridge University Press.

Haag, Rudolf (1992), Local Quantum Physics. NY: Springer.

Halvorson, Hans, and Rob Clifton (2000), "Generic Bell correlation between arbitrary local algebras in quantum field theory", Journal of Mathematical Physics 41: 1711-1717.

Horuzhy, Sergei (1988), Introduction to Algebraic Quantum Field Theory. Dordrecht: Kluwer.

Kadison, Richard V. (1970), "Some analytic methods in the theory of operator algebras", in C. T. Taam (ed.), Lectures in Modern Analysis and Applications, Vol. II. NY: Springer, 8-29.

Kay, Bernard (1979), "A uniqueness result in the Segal-Weinless approach to linear Bose fields", Journal of Mathematical Physics 20: 1712-1713.

Malament, David (1996), "In defense of dogma: Why there cannot be a relativistic quantum mechanics of (localizable) particles", in Rob Clifton (ed.), Perspectives on Quantum Reality. Dordrecht: Kluwer, 1-10.

Petz, Dénes (1990), An Invitation to the Algebra of Canonical Commutation Relations. Leuven University Press.

Redhead, Michael (1995a), "More ado about nothing", Foundations of Physics 25: 123-137.

Redhead, Michael (1995b), "The vacuum in relativistic quantum field theory", in David Hull, Micky Forbes, and Richard M. Burian (eds.), PSA 1994, v. 2. East Lansing, MI: Philosophy of Science Association, 77-87.

Saunders, Simon (1992), "Locality, complex numbers, and relativistic quantum theory", in David Hull, Micky Forbes, and Kathleen Okruhlik (eds.), PSA 1992, v. 1: East Lansing, MI: Philosophy of Science Association, 365-380.

Segal, Irving E. (1964), "Quantum fields and analysis in the solution manifolds of differential equations", in William T. Martin and Irving E. Segal, (eds.), Proceedings of a Conference on the Theory and Applications of Analysis in Function Space. Cambridge: MIT Press, 129-153.

Segal, Irving E., and Roe W. Goodman (1965), "Anti-locality of certain Lorentz-invariant operators", Journal of Mathematics and Mechanics 14: 629-638.

Summers, Stephen J., and Reinhard Werner (1985), "The vacuum violates Bell's inequalities", Physics Letters 110A: 257-259. 